\title{
A Randomized Comparative Study between High-Intensity and Low-Level Laser Therapy in the Treatment of Chronic Nonspecific Low Back Pain
}

\author{
Walid Kamal Abdelbasset $\mathbb{D}^{1,2}$ Gopal Nambi ${ }^{10},{ }^{1}$ Saud F. Alsubaie ${ }^{(D)},{ }^{1}$ \\ Ahmed M. Abodonya $(1),{ }^{3,4}$ Ayman K. Saleh $\left(1,{ }^{4,5}\right.$ Nahla N. Ataalla $(1),{ }^{6}$ Ahmed A. Ibrahim $(1), 7$

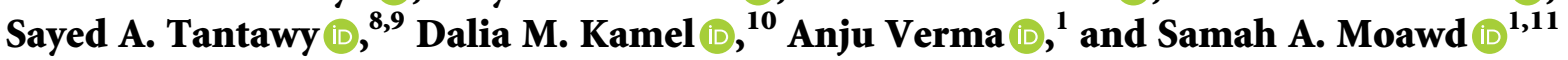 \\ ${ }^{1}$ Department of Health and Rehabilitation Sciences, College of Applied Medical Sciences, Prince Sattam Bin Abdulaziz University, \\ Alkharj, Saudi Arabia \\ ${ }^{2}$ Department of Physical Therapy, Kasr Al-Aini Hospital, Cairo University, Giza, Egypt \\ ${ }^{3}$ Department of Anesthesia and Intensive Care, Faculty of Medicine, Al-Azhar University, Cairo, Egypt \\ ${ }^{4}$ Department of Surgery, College of Medicine, Prince Sattam Bin Abdulaziz University, Alkharj, Saudi Arabia \\ ${ }^{5}$ Department of Orthopedic, Faculty of Medicine for Girls, Al-Azhar University, Cairo, Egypt \\ ${ }^{6}$ Department of Radiological Sciences and Medical Imaging, College of Applied Medical Sciences, \\ Prince Sattam Bin Abdulaziz University, Alkharj, Saudi Arabia \\ ${ }^{7}$ Department of Physical Therapy, College of Applied Medical Sciences, Hai'l University, Hai'l, Hail, Saudi Arabia \\ ${ }^{8}$ Department of Physiotherapy, Center of Radiation Oncologye Nuclear Medicine, Cairo University, Giza, Egypt \\ ${ }^{9}$ Department of Physiotherapy for Integumentary Problems, Faculty of Physical Therapy, Deraya University, Menia, Egypt \\ ${ }^{10}$ Department of Physical Therapy for Women's Health, Faculty of Physical Therapy, Cairo University, Giza, Egypt \\ ${ }^{11}$ Department of Physical Therapy for Cardiovascular/Respiratory Disorder and Geriatrics, Faculty of Physical Therapy, \\ Cairo University, Giza, Egypt
}

Correspondence should be addressed to Walid Kamal Abdelbasset; walidkamal.wr@gmail.com

Received 24 March 2020; Revised 1 September 2020; Accepted 14 October 2020; Published 29 October 2020

Academic Editor: Antonella Fioravanti

Copyright (C) 2020 Walid Kamal Abdelbasset et al. This is an open access article distributed under the Creative Commons Attribution License, which permits unrestricted use, distribution, and reproduction in any medium, provided the original work is properly cited.

Objectives. Chronic nonspecific low back pain (chronic nsLBP) is one of the most common musculoskeletal disorders leading to disabilities and physical inactivity. Laser therapy was used in chronic nsLBP treatment; however, no previous studies have assessed the impacts of high-intensity laser therapy (HILT) versus low-level laser therapy (LLLT) on chronic nsLBP. This study compared the effects of HILT versus LLLT on individuals suffering from chronic nsLBP. Methods. The study was a randomized control trial. Sixty individuals with chronic nsLBP were enrolled in this study between May and November 2019. All participants were clinically diagnosed with chronic nsLBP. They were assigned randomly into three groups, 20 in each group. The first group received a program of LLLT, the second group received a program of HILT, and the third did not receive laser therapy (control group). Pain severity, disability, lumbar mobility, and quality of life were assessed before and after 12-week intervention. Results. Both LLLT and HILT groups showed a significant improvement of the Oswestry Disability Index (ODI), visual analogue scale (VAS), lumbar range of motion (ROM), and European Quality of Life (EuroQol) scores $(p>0.05$ ), while the control group did not show significant changes $(p>0.05)$. Comparison among the three study groups postintervention showed significant differences in the outcome measures $(p>0.05)$, while comparison between the LLLT and HILT groups showed nonsignificant differences $(p>0.05)$. Conclusion. There are no different influences of LLLT versus HILT on chronic nsLBP patients. Both LLLT and HILT reduce pain and disability and improve lumbar mobility and quality of life in chronic nsLBP patients. 


\section{Introduction}

Chronic nonspecific low back pain (chronic nsLBP) is very common and prevalent among the population worldwide. It is identified as pain remaining more than three months without unknown specific pathophysiology [1]. The incidence of a lifetime of chronic nsLBP is approximated to $84.8 \%$ [2]. Chronic nsLBP results in psychological and functional complications and disturbs the quality of life (QoL) $[3,4]$. The conventional intervention of chronic LBP includes medical and nonmedical treatment $[1,5]$.

The underlying causes of the common LBP are exactly undefined [6]. Yearly, ninety-one billion in medical expenditures are spent on back pain with an extra fifty billion indicating indirect costs owing to loss of productivity and payments for disability benefit [7]. One of the significant etiologies of morbidity in adults is LBP [8]. Two-thirds of the individuals are approximately affected several times throughout their lives [9]. It usually causes impairment in the QoL in patients with musculoskeletal system issues [10]. Initial aims of the LBP treatment are to decrease pain, allow patients to continue their activity daily live (ADL), and to improve QoL [5].

Low-level laser therapy (LLLT) is frequently utilized by multiple medicinal sections worldwide; however, the Food and Drug Administration (FDA) has not approved of its indication [11]. The laser emits a monochromatic, nonionizing, noninvasive, polarized electromagnetic, and extremely focused light radiation. LLLT is an effective physiotherapy modality in the management of various musculoskeletal dysfunctions due to its anti-inflammatory, muscle relaxant, analgesic, ligament repairing, tissue revolution, fibroblast exploding, and biostimulant effects $[12,13]$. LLLT is usually used to control chronic and acute pain [14]. Another form of laser therapy for controlling musculoskeletal pain is high-intensity laser therapy (HILT). This modality is convenient, noninvasive, and painless, improves joint mobility, stimulates efficiently deepen tissue, and provides anti-inflammation, analgesics, and other useful influences $[15,16]$. A previous study found that intense pain can be reduced using pulsed HILT [17]. In addition, it exhibits anti-inflammatory, antiedematous, and analgesic effects for a patient with a pain problem [18]. Moreover, it is able to stimulate areas that are difficult to reach with the LLLT, such as the large and/or deep joints [19].

Several studies found that LLLT is an effective modality for controlling chronic nsLBP [20,21], whereas others found that HILT is a safe and useful modality in reducing pain in patients with chronic nsLBP $[22,23]$. As well, more energy may be transmitted using HILT into the exposed tissues when compared with LLLT [19]. To the best of knowledge, no previous studies have assessed the impacts of HILT versus LLLT on chronic nsLBP. Consequently, the current study aimed to evaluate the different effects of HILT and LLLT on chronic nsLBP, hypothesizing no differences between both forms of laser therapy.

\section{Materials and Methods}

2.1. Subjects. This randomized comparative study was conducted in the outpatient physical therapy clinic between
May and November 2019. Sixty chronic nsLBP patients were recruited from the orthopedic outpatient clinic at Prince Sattam Bin Abdulaziz University Hospital. All participants were clinically diagnosed with chronic nsLBP using clinical, laboratory, and radiological examinations. They were assigned randomly into three groups using Dacima's Randomization Software, 20 in each group. The first group received a program of low-level laser therapy (LLLT group) plus home exercise training, the second group received a program of high-intensity laser therapy (HILT group) plus home exercise training, and the third group conducted only home exercise training without laser therapy (control group). Randomization was performed by a blinded physiotherapist who was unaware of baseline evaluations. The inclusion criteria were as follows: (a) history of low back pain lasting more than 3 months; (b) age of 25-40 years; and (c) ability to comply with any of the randomly selected treatment programs. The exclusion criteria were neurological defects, abnormal laboratory findings, fracture, spondylosis, spinal stenosis, inflammatory disease, infectious diseases, mental conditions, prior spinal surgery, and pregnancy. Subjects who have received any type of treatment for back pain in the last three months were also removed. Consent forms were obtained from each participant before initiating the study program. This randomized trial has been ethically approved by the Local Ethics Committee of the Physical Therapy Department, Prince Sattam Bin Abdulaziz University (no. RHPT/019/035) according to the guidelines of the 1964 Helsinki Declaration.

2.2. Sample Size Estimation. The study power was determined by G $*$ Power 3.1 software with $\alpha=0.05, Z=1.95$, and power $=0.80$. The sample size of 17 participants was required for each group. Hence, this randomized trial has included 20 subjects to account for the dropout rate.

2.3. Initial Assessment. Disability, pain severity, range of motion (ROM) of the lumbar flexion, and QoL were examined before and after the treatment program in the three groups by blinded orthopedists that were unaware of the group assignments.

2.4. Oswestry Disability Index (ODI). The ODI is a valid and reliable instrument used to assess the disability level in LBP patients. It comprises 10 items including pain, sleep, walking, carrying, self-help and private life ability, standing, sitting, social and sexual life, and traveling. Every item is scored $0-5$ points depending on the real position, with a total score from 0 to 50 , and earnest dysfunction means a high score [24].

2.5. Visual Analogue Scale (VAS). VAS is a valid pain intensity measure. Each patient was instructed to place a sign in a $10 \mathrm{~cm}$ long part based on the pain severity. It scores $0-10,0$ points to no pain, and 10 points to severe pain. It is commonly utilized to assess lumbosacral pain and it is radiating in the lower limb [25]. 
2.6. Lumbar Flexion Range of Motion (ROM). The range of motion of the lumbar flexion was assessed using a valid and reliable modified Schober test. This measure is one of the most common measures used to assess the lumbar ROM [26].

2.7. Quality of Life (QoL) Questionnaire. The QoL has been measured using EuroQol-5 Dimensions-3 Levels (EQ-5D-3L) [27]. Dimensions include self-care, mobility, pain/discomfort, usual activities, and anxiety/depression with a $0-100$ rating scale, where 0 reveals the worst possible health state and 100 is the best possible health state. It is a homogenized tool for determining health outcomes. EQ-5D-3L is a valid and reliable tool, does not necessitate an effort or prolonged timing for filling it, and was recognized in several prior studies to assess QoL in extensive ranges of health conditions. The EuroQol group commended a valid Arabic adaptation of the EQ-5D scale to examine the quality of life. Each dimension involves 3 levels (nothing, slight/moderate problems, and severe/extreme problems) [28].

2.8. Intervention. All 60 patients have received home exercise training including strengthening exercise for back and abdominal muscles and stretching exercise for back muscles, at least 2 times per week for 12 weeks. Patients have been instructed to not receive painkillers or nonsteroidal antiseditious medications during the study period [29].

2.9. LLLT Protocol. The diode laser device (Chattanooga Group, USA) of gallium-aluminum-arsenide (GaAlAs, infrared laser) with an $850 \mathrm{~nm}$ wavelength, $800 \mathrm{~mW}$ power, and constant wave with $1 \mathrm{~cm}$ spot size was utilized for the treatment procedures. The device of laser was used with a $1 \mathrm{KHz}$ pulsed frequency and duty cycle of $80 \%$ with an average energy density of $50 \mathrm{~J} / \mathrm{cm}^{2}$ for chronic nsLBP for $30 \mathrm{~min} /$ session with a total energy of approximately $1200 \mathrm{~J}, 2$ sessions per week for 12 weeks (24 sessions). Each patient has been instructed to lie prone or modified side-lying position during the session. Both the therapist and the participant wore protective goggles for safety during the treatment time.

2.10. HILT Protocol. The device of BTL-6000 HILT utilizes a gallium-arsenide diode laser (BTL-6000 laser) and was set manually to biostimulating mode with $12 \mathrm{~W}$ power, $150 \mathrm{~J} / \mathrm{cm}^{2}$ energy, $1064 \mathrm{~nm}$ wavelength, and $1 \mathrm{~cm}^{2}$ patch radiation diameter zone. Under these cases, the laser was performed with a constant movement. During a session, total energy applied to subjects measured $1200 \mathrm{~J}, 2$ sessions per week for 12 weeks (24 sessions). Application time lasted for $15 \mathrm{~min}$ during each session. Each patient has been instructed to lie prone or modified side-lying position during the session. The device probe was vertically situated and moved horizontally on the affected area.
2.11. Statistical Analysis. All outcome measures were presented in the form of means \pm standard deviations. Changes of the variables among the three groups were assessed using one-way ANOVA, while pre-post changes within each group were assessed using a paired $t$-test. Statistically, all variables were analyzed using SPSS (v.25, IBM Corp., Armonk, NY, USA). The significant level was considered at $p$ value ${ }^{<} 0.05$.

\section{Results}

All the sixty patients have completed the twelve weeks of the study program as described in the flowchart of the study (Figure 1). Baseline characteristics showed no significant differences among the three study groups (age, $p=0.651$; gender, $p=0.836$; BMI, $p=0.642$; LBP duration $p=0.961$; education level, $p=0.913$; occupation, $p=0.981$; and marital status, $p=0.506$ ) as demonstrated in Table 1 . Outcome measures showed no significant differences between groups preintervention (ODI, $p=0.726$; VAS, $p=0.534$; lumbar ROM, $p=0.623$; and EuroQol, $p=0.463$ ). LLLT showed significant improvements of ODI, VAS, lumbar ROM, and EuroQol scores postintervention $(p<0.001)$ and also HILT showed significant improvements of ODI, VAS, lumbar ROM, and EuroQol scores postintervention $(p<0.001)$, while the control group showed nonsignificant changes $(p>0.05)$ as shown in Table 2 . Comparison between the three study groups postintervention showed a significant difference in all outcome measures $(p<0.001)$, while comparison between the LLLT and HILT groups postintervention showed nonsignificant differences $(p>0.05)$ through post hoc analysis as demonstrated in Table 2.

\section{Discussion}

This study aimed to evaluate the effects of HILT versus LLLT on patients with chronic nsLBP hypothesizing that laser therapy whether LLLT or HILT may relieve pain and improve quality of life in chronic nsLBP patients. The findings of this study showed that both LLLT and HILT result in a significant improvement of ODI, VAS, lumbar ROM, and EuroQol scores at the end of the study intervention.

The analgesic and anti-inflammatory effects of LLLT are associated with elevating of pain threshold and inhibiting the transmission of A- $\delta$ and $C$ fibers that combined with suppressed peripheral nociceptors, increased hydroxyindoleacetics, decreased inflammatory cytokines such as IL- $1 \beta$, IL-8, and TNF- $\alpha$, and reduced prostaglandin levels [30]. Moreover, LLLT inhibits pain through enhancing the secretions of peripheral endogenous opioids [31]. It was reported that infrared laser GaAlAs with a wavelength of $808 \mathrm{~nm}$ is a safe and effective modality for decreasing the disability and reducing the severity of pain in LBP patients [32].

Reducing the level of C-reactive protein with laser irradiation is also associated with antiseditious influences of low-level laser therapy [33]. This study utilizes a highintensity level therapy instrument, which is a novel manner of laser therapy that recently began. This modality of laser intervention demonstrates similar influences of 


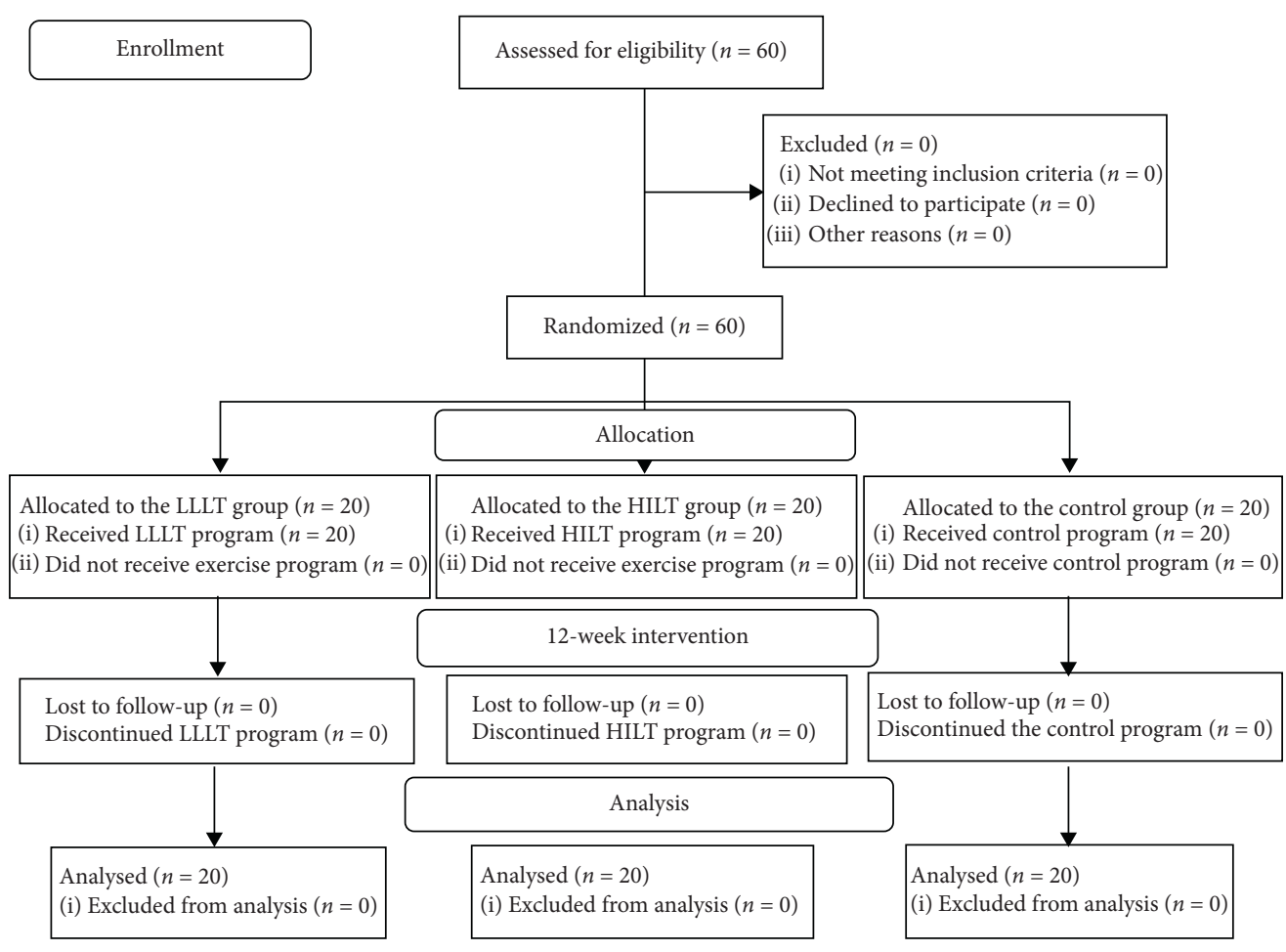

FIGURE 1: The flowchart of the study.

TABLE 1: Baseline characteristics of three study groups.

\begin{tabular}{|c|c|c|c|c|}
\hline Variables & LLLT $(n=20)$ & HILT $(n=20)$ & Control $(n=20)$ & $p$ value \\
\hline Age, years & $32.4 \pm 3.7$ & $33.6 \pm 4.5$ & $32.8 \pm 4.2$ & 0.651 \\
\hline Gender, M/F & $14 / 6$ & $13 / 7$ & $15 / 5$ & 0.836 \\
\hline BMI, $\mathrm{kg} / \mathrm{m}^{2}$ & $25.4 \pm 2.9$ & $25.9 \pm 2.5$ & $26.2 \pm 2.7$ & 0.642 \\
\hline LBP duration, months & $8.12 \pm 3.5$ & $7.94 \pm 3.2$ & $8.25 \pm 3.7$ & 0.961 \\
\hline \multicolumn{5}{|l|}{ Education level, $n(\%)$} \\
\hline Academic education & $6(30)$ & $7(35)$ & $5(25)$ & \multirow{3}{*}{0.913} \\
\hline Middle education & $10(50)$ & $8(40)$ & $9(45)$ & \\
\hline Primary education or less & $4(20)$ & $5(25)$ & $6(30)$ & \\
\hline \multicolumn{5}{|l|}{ Occupation, $n$ (\%) } \\
\hline Employed & $10(50)$ & $9(45)$ & $11(55)$ & \multirow{3}{*}{0.981} \\
\hline Unemployed & $4(20)$ & $5(25)$ & $4(20)$ & \\
\hline Workers & $6(30)$ & $6(30)$ & $5(25)$ & \\
\hline \multicolumn{5}{|l|}{ Marital status, $n(\%)$} \\
\hline Married & $16(80)$ & $18(90)$ & $15(75)$ & \multirow{2}{*}{0.506} \\
\hline Single & $4(20)$ & $2(10)$ & $5(25)$ & \\
\hline
\end{tabular}

Variables are presented as means \pm standard deviations; significant at $p$ value $<0.05$; M: males; F; females; BMI: body mass index; ODI: Oswestry Disability Index; VAS: visual analogue scale; ROM: range of motion; EuroQol: European Quality of Life score.

the common laser while with more focused and powerful photoenergy effects, with a more sufficient concentration of endogenous chromophores throughout the treatment program depending on the wavelength. The proliferation of the laser irradiation through the body realizes easily deep penetration, achieves the spread out in the tissue, enhances the oxidative response of mitochondria, generates ATP, RNA, and DNA, enhances photobiological impacts on the affected tissues, stimulates collagen production of the muscle tendons, and accordingly improves the status of chronic nsLBP patients and their daily life activities [19, 34].
Additionally, the study results showed that patients in laser groups displayed a higher degree of improvement utilizing ODI, VAS, lumbar flexion ROM, and quality of life when compared with the control group. HILT of analgesic and detumescence facilitates inflammation resolution of the local issue, improves circulation, reduces LBP, and improves the recovery of function, coinciding with previous study results. HILT conducts actual-duration, complex, larger scale, deeper penetration, and more dose behavior on the body's affected tissues compared to low-level lasers. The laser therapy also improves disease conditions, which play a key role in relieving pain and function recovery [35]. 
TABLE 2: The differences in mean values within and between groups pre- and postintervention.

\begin{tabular}{|c|c|c|c|c|}
\hline Variables & Preintervention & Postintervention & Mean difference $(95 \% \mathrm{CI})$ & $p$ value \\
\hline \multicolumn{5}{|l|}{ ODI } \\
\hline LLLT & $36.5 \pm 12.7$ & $17.8 \pm 6.4$ & 18.75 (16.8 to 20.6$)$ & $<0.001$ \\
\hline HILT & $37.3 \pm 11.3$ & $18.5 \pm 7.2$ & $18.8(16.77$ to 20.8$)$ & $<0.001$ \\
\hline Control & $36.2 \pm 12.3$ & $34.6 \pm 11.8$ & $1.6(-1.4$ to 5.3$)$ & 0.395 \\
\hline$p$ value & 0.726 & $<0.001$ & - & - \\
\hline \multicolumn{5}{|l|}{ VAS } \\
\hline LLLT & $6.5 \pm 1.7$ & $3.4 \pm 0.9$ & $3.1(2.5$ to 3.6$)$ & $<0.001$ \\
\hline HILT & $6.3 \pm 1.9$ & $3.5 \pm 0.8$ & $2.8(2.4$ to 3.2$)$ & $<0.001$ \\
\hline Control & $6.6 \pm 1.6$ & $5.9 \pm 1.8$ & $0.7(0.19$ to 1.2$)$ & 0.293 \\
\hline$p$ value & 0.534 & $<0.001$ & - & - \\
\hline \multicolumn{5}{|c|}{ Lumbar ROM } \\
\hline LLLT & $17.3 \pm 2.2$ & $20.4 \pm 1.7$ & $-3.1(-2.5$ to -3.6$)$ & $<0.001$ \\
\hline HILT & $16.9 \pm 2.1$ & $20.6 \pm 1.8$ & $-3.7(-2.8$ to -4.1$)$ & $<0.001$ \\
\hline Control & $17.6 \pm 2.5$ & $17.9 \pm 2.4$ & $-0.3(-0.39$ to -0.21$)$ & 0.082 \\
\hline$p$ value & 0.623 & $<0.001$ & - & - \\
\hline \multicolumn{5}{|c|}{ Total EuroQol } \\
\hline LLLT & $32.5 \pm 8.4$ & $72.8 \pm 9.7$ & $-40.3(-45.6$ to -36.7$)$ & $<0.001$ \\
\hline HILT & $35.7 \pm 9.6$ & $74.3 \pm 10.4$ & $-38.6(-42.3$ to -35.4$)$ & $<0.001$ \\
\hline Control & $35.4 \pm 8.8$ & $38.6 \pm 9.2$ & $-3.2(-5.3$ to -1.4$)$ & 0.079 \\
\hline$p$ value & 0.463 & $<0.001$ & - & - \\
\hline
\end{tabular}

Variables are presented as means \pm standard deviations; significant at $p$ value $<0.05$; ODI: Oswestry Disability Index; VAS: visual analogue scale; ROM: range of motion; EuroQol: European Quality of Life score.

Although this randomized study provides important findings, it has some limitations. Because the study has included only chronic nsLBP patients with 25-40 years, it cannot be generalized to other ages. Another limitation is the lack of long-term follow-up of the study (6 months to one year postintervention). Future studies may compare laser therapy and other modalities of electrotherapy in the rehabilitation field.

\section{Conclusions}

Based on the study outcomes, there are no different effects of LLLT versus HILT in the treatment of chronic nsLBP. Both LLLT and HILT reduce pain and disability and improve lumbar ROM and quality of life in patients with chronic nsLBP.

\section{Data Availability}

The data involved in this study are available from the corresponding author upon request, and privacy-related parts of the patient will not be provided.

\section{Conflicts of Interest}

The authors declare that they have no conflicts of interest.

\section{Acknowledgments}

This project was supported by the Deanship of Scientific Research at Prince Sattam Bin Abdulaziz University under the research project no. 2020/03/11877. The authors would like to thank all patients who participated in this study.

\section{References}

[1] R. Chou, A. Qaseem, V. Snow et al., "Diagnosis and treatment of low back pain: a joint clinical practice guideline from the American college of physicians and the American pain society," Annals of Internal Medicine, vol. 147, no. 7, pp. $478-491,2007$.

[2] B. M. Mohseni, F. Ehsani, H. Behtash, and M. Ghanipour, "Occupational low back pain in primary and high school teachers: prevalence and associated factors," Journal of $\mathrm{Ma}$ nipulative and Physiological Therapeutics, vol. 37, no. 9, pp. 702-708, 2014

[3] A. F. Mannion, M. Müntener, J. Dvorak, and S. Taimela, "Comparison of three active therapies for chronic low back pain: results of a randomized clinical trial with one-year follow-up," Rheumatology, vol. 40, no. 7, pp. 772-778, 2001.

[4] J. Rainville, E. C. Hartigan, C. J. Martinez, M. Limke, C. Jouve, and M. Finno, "Exercise as a treatment for chronic low back pain," The Spine Journal, vol. 4, no. 1, pp. 106-115, 2004.

[5] R. Chou and L. H. Huffman, "Nonpharmacologic therapies for acute and chronic low back pain: a review of the evidence for an American pain society/American college of physicians clinical practice guideline," Annals of Internal Medicine, vol. 147, no. 7, pp. 492-504, 2007.

[6] M. W. Van Tulder, B. W. Koes, J. F. Metsemakers, and L. M. Bouter, "Chronic low back pain in primary care: a prospective study on the management and course," Family Practice, vol. 15, no. 2, pp. 126-132, 1998.

[7] A. D. Furlan, F. Yazdi, A. Tsertsvadze et al., "A systematic review and meta-analysis of efficacy, cost-effectiveness, and safety of selected complementary and alternative medicine for neck and low-back pain," Evidence-Based Complementary and Alternative Medicine, vol. 2012, Article ID 953139, 11 pages, 2012.

[8] N. R. Yousefi, E. Schonstein, K. Heidari et al., "Low level laser therapy for nonspecific low back pain," Cochrane Database of Systematic Reviews, vol. 4, no. 2, Article ID CD005107, 2008. 
[9] G. E. Djavid, R. Mehrdad, M. Ghasemi, H. Hasan-Zadeh, A. Sotoodeh-Manesh, and G. Pouryaghoub, "In chronic low back pain, low level laser therapy combined with exercise is more beneficial than exercise alone in the long term: a randomised trial," Australian Journal of Physiotherapy, vol. 53, no. 3, pp. 155-160, 2007.

[10] Z. Unlu, S. Tasci, S. Tarhan, Y. Pabuscu, and S. Islak, "Comparison of 3 physical therapy modalities for acute pain in lumbar disc herniation measured by clinical evaluation and magnetic resonance imaging," Journal of Manipulative and Physiological Therapeutics, vol. 31, no. 3, pp. 191-198, 2008.

[11] J. N. Awad and R. Moskovich, "Lumbar disc herniations," Clinical Orthopaedics and Related Research, vol. 443, pp. 183-197, 2006.

[12] L. Brosseau, G. Wells, S. Marchand et al., "Randomized controlled trial on low level laser therapy (LLLT) in the treatment of osteoarthritis (OA) of the hand," Lasers in Surgery and Medicine, vol. 36, no. 3, pp. 210-219, 2005.

[13] S. A. Tantawy, W. K. Abdelbasset, D. M. Kamel, and S. M. Alrawaili, "A randomized controlled trial comparing helium-neon laser therapy and infrared laser therapy in patients with diabetic foot ulcer," Lasers in Medical Science, vol. 33, no. 9, pp. 1901-1906, 2018.

[14] S. A. Tantawy, W. K. Abdelbasset, D. M. Kamel, S. M. Alrawaili, and S. F. Alsubaie, "Laser photobiomodulation is more effective than ultrasound therapy in patients with chronic nonspecific low back pain: a comparative study," Lasers in Medical Science, vol. 34, no. 4, pp. 793-800, 2019.

[15] N. O. Pekyavas and G. Baltaci, "Short-term effects of highintensity laser therapy, manual therapy, and Kinesio taping in patients with subacromial impingement syndrome," Lasers in Medical Science, vol. 31, no. 6, pp. 1133-1141, 2016.

[16] M. S. M. Alayat, A. A. Mohamed, O. F. Helal, and O. A. Khaled, "Efficacy of high-intensity laser therapy in the treatment of chronic neck pain: a randomized double-blind placebo-control trial," Lasers in Medical Science, vol. 31, no. 4, pp. 687-694, 2016.

[17] A. Santamato, V. Solfrizzi, F. Panza et al., "Short-term effects of high-intensity laser therapy versus ultrasound therapy in the treatment of people with subacromial impingement syndrome: a randomized clinical trial," Physical Therapy, vol. 89, no. 7, pp. 643-652, 2009.

[18] R. Saggini, R. G. Bellomo, and F. Cancelli, "Hilterapia and chronic ankle pain syndromes," Abstract from Energy for Health;International journal of information and scientific culture, vol. 3, no. 3, pp. 22-25, 2009.

[19] A. Zati and A. Valent, "Physical therapy: new technologies in rehabilitation medicine (translated to english)," Edizioni Minerva Medica, vol. 2006, pp. 162-185, 2006.

[20] G. Glazov, M. Yelland, and J. Emery, "Low-level laser therapy for chronic non-specific low back pain: a meta-analysis of randomised controlled trials," Acupuncture in Medicine, vol. 34, no. 5, pp. 328-341, 2016.

[21] Z. Huang, J. Ma, J. Chen, B. Shen, F. Pei, and V. B. Kraus, "The effectiveness of low-level laser therapy for nonspecific chronic low back pain: a systematic review and meta-analysis," Arthritis Research \& Therapy, vol. 17, p. 360, 2015.

[22] P. Fiore, F. Panza, G Cassatella et al., "Short-term effects of high-intensity laser therapy versus ultrasound therapy in the treatment of low back pain: a randomized controlled trial," European Journal of Physical and Rehabilitation Medicine, vol. 47, no. 3, pp. 367-373, 2011.
[23] M. Gocevska, E. Nikolikj-Dimitrova, and C. GjerakaroskaSavevska, "Effects of high-intensity laser in treatment of patients with chronic low back pain," Open Access Macedonian Journal of Medical Sciences, vol. 7, no. 6, pp. 949-954, 2019.

[24] C. P. Lee, T. S. Fu, C. Y. Liu, and C. I. Hung, "Psychometric evaluation of the oswestry disability index in patients with chronic low back pain: factor and mokken analyses," Health Qual Life Outcomes, vol. 15, no. 1, p. 192, 2017.

[25] I. S. K. Thong, M. P. Jensen, J. Miró, and G. Tan, “The validity of pain intensity measures: what do the NRS, VAS, VRS, and FPS-R measure?" Scandinavian Journal of Pain, vol. 18, no. 1, pp. 99-107, 2018.

[26] A. Rezvani, O. Ergin, I. Karacan, and M. Oncu, "Validity and reliability of the metric measurements in the assessment of lumbar spine motion in patients with ankylosing spondylitis," Spine, vol. 37, no. 19, pp. E1189-E1196, 2012.

[27] The EuroQol Group, "EuroQol-a new facility for the measurement of health-related quality of life," Health Policy, vol. 16, pp. 199-208, 1990.

[28] W. K. Abdelbasset, S. F. Alsubaie, S. A. Tantawy, Abo Elyazed TI, and A. A. Elshehawy, "A cross-sectional study on the correlation between physical activity levels and health related quality of life in community-dwelling middle-aged and older adults," Medicine, vol. 98, no. 11, Article ID e14895, 2019.

[29] A. Stergioulas, "Low-power laser treatment in patients with frozen shoulder: preliminary results," Photomedicine and Laser Surgery, vol. 26, no. 2, pp. 99-105, 2008.

[30] S. Gopal Nambi, W. Kamal, J. George, and E. Manssor, "Radiological and biochemical effects (CTX-II, MMP-3, 8, and 13) of low-level laser therapy (LLLT) in chronic osteoarthritis in Al-Kharj, Saudi Arabia," Lasers in Medical Science, vol. 32, no. 2, pp. 297-303, 2017.

[31] L. Kholoosy, D. Elyaspour, M. R. Akhgari, Z. Razzaghi, Z. Khodamardi, and M. Bayat, "Evaluation of the therapeutic effect of low level laser in controlling low back pain: a randomized controlled trial," Journal of Lasers in Medical Sciences, vol. 11, no. 2, pp. 120-125, 2020.

[32] S. Hagiwara, H. Iwasaka, A. Hasegawa, and T. Noguchi, "Preirradiation of blood by gallium aluminum arsenide $(830 \mathrm{~nm})$ low-level laser enhances peripheral endogenous opioid analgesia in rats," Anesthesia \& Analgesia, vol. 107, no. 3, pp. 1058-1063, 2008.

[33] A. C. Freitas, A. L. Pinheiro, P. Miranda, F. A. Thiers, and A. L. Vieira, "Assessment of anti-inflammatory effect of 830nm laser light using C-reactive protein levels," Brazilian Dental Journal, vol. 12, no. 3, pp. 187-190, 2001.

[34] A. R. Kheshie, M. S. M. Alayat, and M. M. E. Ali, "Highintensity versus low-level laser therapy in the treatment of patients with knee osteoarthritis: a randomized controlled trial," Lasers in Medical Science, vol. 29, no. 4, pp. 1371-1376, 2014.

[35] U. Dundar, U. Turkmen, H. Toktas, A. M. Ulasli, and O. Solak, "Effectiveness of high-intensity laser therapy and splinting in lateral epicondylitis; a prospective, randomized, controlled study," Lasers in Medical Science, vol. 30, no. 3, pp. 1097-1107, Sci2015. 